\title{
On the Impact of Selfish Behaviors in Wireless Packet Scheduling
}

\author{
Zhen Kong \\ Department of EEE \\ The University of Hong Kong \\ Hong Kong, China \\ zkong@eee.hku.hk
}

\author{
Yu-Kwong Kwok \\ Department of ECE \\ Colorado State University \\ Fort Collins, CO 80523, USA \\ Ricky.Kwok@colostate.edu
}

\author{
Jiangzhou Wang \\ National Mobile Communications Lab \\ Southeast University \\ Nanjing, 210096, China
}

\begin{abstract}
In many practical scenarios, wireless devices are autonomous and thus, may exhibit non-cooperative behaviors due to self-interests. For instance, a wireless user may report bogus channel information to gain resource allocation advantages. Such non-cooperative behaviors are practicable as the device's software could be modified by the user. In this paper, we first analyze the impact of these rationally selfish behaviors on the performance of packet scheduling algorithms in time-slotted wireless networks. Using a mixed strategy game theoretic model, we show that the traditional Maximum Rate packet scheduling algorithm can lead non-cooperative users to undesirable Nash equilibriums, in which the wireless channels are used inefficiently. By using repeated game to enforce cooperation, we further propose a novel game theoretic approach that can lead to an efficient equilibrium.
\end{abstract}

Keywords - packet scheduling; selfish behavior; game theory; non-cooperative wireless networks

\section{INTRODUCTION}

In a centralized infrastructure based wireless network, packet scheduling is a very important component for managing the precious radio resource while satisfying users' QoS requirements. Specifically, in a traditional downlink packet scheduling process, the wireless users will report their channel conditions, such as SINR or maximal achievable data rate, to the scheduler located at the base station (BS). Then the scheduler can select some users' packets and allocate radio resource, such as power and frequency bandwidth, to them for transmission according to some scheduling policies, such as Maximum Rate [1] and proportional fairness [2].

Usually, these scheduling algorithms are based on the assumption that the wireless users in the system will cooperate with each others, comply with the predefined scheduling algorithm, and honestly report their real channel conditions to the scheduler. Then the wireless user will accept the scheduling results passively. However, in many practical scenarios, the users are autonomous and thus, may exhibit non-cooperative behaviors due to self-interests and try to gain their own advantages without regard to the overall system performance [3]. For example, a user experiencing bad channel condition might find out that if it honestly reports his channel condition to the scheduler, it may not be scheduled or just be assigned a low data rate. Consequently, with a rationally selfish motivation, such user might report a bogus channel condition so as to get a higher probability to be scheduled for transmission or get a higher data rate. Though this non-cooperative behavior could increase the throughput for this selfish user, it may lead to inefficient resource utilization for the whole system. Such non-cooperative behaviors are practicable because the wireless device's software could be modified by the user [4]. Consequently, whether the traditional packet scheduling algorithms are still effective in allocating resource in non-cooperative environment is in doubt and needs to be scrutinized carefully.

Due to the increasing interests and capabilities for mobile users to deviate from legitimated protocols and algorithms so as to increase their own benefits, the research for selfish behavior has received considerable attention for various aspects of wireless networks in recent years, such as wireless routing [5][6], power control [7][8], MAC misbehavior in WLAN [9][10], and even Denial of Service attack [11][12], which can be considered as an extreme case of misbehavior. To the best of our knowledge, the impact of non-cooperative behaviors on wireless packet scheduling is a relatively unexplored research problem.

In this paper, we focus on the issue of downlink packet scheduling for selfish and rational users in a TDMA wireless network, in which each user wishes to selfishly choose its transmission rate in such a way so as to maximize its utility. However, the strategy chosen by a user may also affect the performances of other users in the network through the related packet scheduling algorithm. Therefore in practice, a rational user will evaluate its achieved data rate, average packet transmission success rate (PTSR), the probability of being scheduled to determine the best strategy for reporting a "best" channel condition to the scheduler, as so to improve its gain. When concerning this interaction, there are several questions to be asked. First of all, what kind of strategy should a user choose in order to maximize its utility? Then, what is the consequent impact of this kind of selfish misbehavior on the system performance and its own benefit? If every user in the network selfishly picks its utility-maximizing strategy, will there be a stable state at which no user can unilaterally improve its utility, e.g. Nash equilibrium (NE)? And if this NE will lead to inefficient radio resource utilization, are there any other methods that can be proposed to improve the performance?

Based on game theory [13], we formulate this problem as a non-cooperative packet scheduling (NPS) game, and set up a novel mixed strategy game model, in which each player has a continuum of actions and seeks to choose its transmission rate to maximize its overall utility, which is defined as the expected data rate. We then deduce the corresponding $\mathrm{NE}$ for this game and find that the NE strategy leads to an allocation that is Pareto inefficient, where the system throughput is much lower than that in normal cooperative situation. Motivated by this observation, 
we formulate a repeated game to enforce cooperation among wireless users, and propose an efficient strategy to increase the throughput performance in a non-cooperative environment.

The remainder of this paper is organized as follows. In Section II, we describe the system model and discuss the impact of selfish behavior on system performances. Then the NPS game is analyzed in Section III. In Section IV, we propose a repeated game theoretic strategy to improve the performances. Finally, we conclude in Section V.

\section{MODEL}

\section{A. System model}

We consider a time-slotted system with a BS serving $N$ wireless users. All users are assumed to be within the same communication range (i.e., each user can overhear any other users). And they always have packets to transmit. The BS transmits in slots with fixed duration, and only one user can be scheduled in one time slot. At the beginning of time slot $t$, each user $i$ measures the downlink channel condition, and return, via a feedback channel, a measured data rate $r_{i}(t)$ to the BS. Then, based on this information, the BS determines which user to transmit its packet at this time slot.

In our analysis, we assume QAM modulation and ideal phase detection are used in a Rayleigh fading channel with bandwidth $W$, and no retransmission is considered. By using adaptive modulation, at time instant $t$, the maximal achievable symbol rate $c_{i}^{0}(t)$ (bit/symbol) for user $i$ can be usually decided by the current channel SNR and the required bit-error-rate (BER), and it can be expressed as [14]:

$$
c_{i}^{0}(t)=\log _{2}\left(1+\frac{-1.5}{\ln \left(5 \cdot P_{b e r}\right)} \cdot \gamma_{i}(t)\right)
$$

where $\gamma_{i}(t)$ is the SNR for the user $i$ at time instant $t$, and $P_{b e r}$ is the required BER for this transmission. Then the corresponding maximal achievable data rate is given by:

$$
r_{i}^{0}(t)=c_{i}^{0}(t) \cdot W
$$

We assume that each user can report its channel information to the BS in an error-free manner. Within such a simple framework, several existing packet scheduling algorithms can be used, such as MR algorithm [1], which is designed to maximize the rate at each slot, as well as the system total rate, by scheduling the user with the largest $r_{i}(t)$ for transmission. In this paper, we focus on the performance of the MR algorithm under a non-cooperative wireless environment.

\section{B. Selfish behavior and its impact}

In a cooperative situation, the feedback rate $r_{i}(t)$ from user $i$ is just the maximum feasible rate $r_{i}^{0}(t)$ that the downlink can support under its current SNR $\gamma_{i}$ to maintain a certain BER or PTSR requirement.

On the other hand, in a non-cooperative environment, a selfish but rational user may report a bogus data rate $r_{i}(t)>r_{i}^{0}(t)$, so as to enhance its opportunity to be chosen for transmission as governed by the scheduling algorithm. Of course, a higher assigned data rate $r_{i}(t)$ may result in a higher BER or lower PTSR under the same SNR $\gamma_{i}$, making its actual rate smaller than the intended value. Nevertheless, a rationally selfish consideration is that the smaller realized rate may be compensated by the rate improvement induced by the increased transmission probability. Consequently, a non-cooperative node could still have the incentive to report a different data rate to the BS so as to increase its own potential payoff.

Let $\operatorname{BER}\left(r_{i}, r_{i}^{0}\right)$ and $\alpha\left(r_{i}, r_{i}^{0}\right)$ respectively be the BER and PTSR for user $i$ when its maximum feasible rate is $r_{i}^{0}$ and feedback data rate is $r_{i}$, where $r_{i}^{0} \in\left\{r^{1}, r^{2}, \cdots, r^{M}\right\}$ and $r_{i} \in\left\{r^{1}, r^{2}, \cdots, r^{M}\right\}$. When there are $L$ bits in one packet, we have

$$
\operatorname{BER}_{i}\left(r_{i}, r_{i}^{0}\right)=0.2 \cdot \exp \left(\log _{2}\left(5 \cdot P_{b e r}\right) \cdot \frac{\left(2^{r_{i}{ }^{0}}-1\right)}{\left(2^{r_{i}}-1\right)}\right)
$$

and

$$
\alpha\left(r_{i}, r_{i}^{0}\right)=\left(1-B E R_{i}\left(r_{i}, r_{i}^{0}\right)\right)^{L}
$$

Since the probability that it can transmit at this time slot is $\operatorname{Pr}\left(r_{i}(t)>r_{j}(t), j \neq i\right)$, the expected rate can be expressed as:

$$
\begin{aligned}
& \mathrm{R}_{i}\left(r_{i}(t)\right)= \\
& \left\{\begin{array}{l}
r_{i}^{0} \cdot \alpha\left(r_{i}^{0}, r_{i}^{0}\right) \cdot \operatorname{Pr}\left(r_{i}(t)=r_{i}^{0}>r_{j}(t), j \neq i\right) \quad \text { cooperative } \\
r_{i} \cdot \alpha\left(r_{i}, r_{i}^{0}\right) \cdot \operatorname{Pr}\left(r_{i}(t)=r_{i}>r_{j}(t), j \neq i\right) \quad \text { noncooperative }
\end{array}\right.
\end{aligned}
$$

In this study, we consider a scenario with $N$ users. And there are $S$ selfish users deliberately deviating from the packet scheduling algorithm. Since we assume all the users are within the same communication range, the selfish user $i$ can overhear the feedbacks of other users and report a bogus rate $r_{i}>r_{j}, j \neq i$.

To show the impact of this kind of selfish behavior, we conduct simulations based on the above model. The entire system bandwidth is $10 \mathrm{MHz}$, and the time slot duration is set to be $100 \mathrm{~ms}$. We let $c_{i} \in\{2,4, \cdots, 10\}$. The wireless channel is modeled as six-path frequency-selective Rayleigh fading channel. Each path is simulated by Clark's fading model and suffers from independent Rayleigh fading. We assume the packet length is 8 bits/packet and the maximum BER requirement is $10^{-5}$.

Fig. 1 shows the impact of selfish behavior on the performance of traditional MR algorithm. There are 8 users. And user 1 deliberately fails to adhere to the algorithm and tries to misbehave, following the selfish model presented above. In cooperative environment, all users will report their maximum feasible data rates to the base station; whereas in non-cooperative environment, user 1 (U1) will report a bogus data rate to the BS and all other users still report their maximum feasible rates honestly. In this simulation, we can see in Fig. 1 that U1 can achieve about $100 \%$ increase in throughput by reporting a higher data rate and behaving selfish. However, as a result of U1 using this selfish strategy, the total throughput would decrease by $30 \%$. Therefore, the existence of selfish behavior in non-cooperative wireless networks will make the throughput performance of packet scheduling algorithm significantly degraded. Most 
importantly, this observation still holds in the network with different sizes, such as those from 2 users to 20 users as shown in Fig.2.

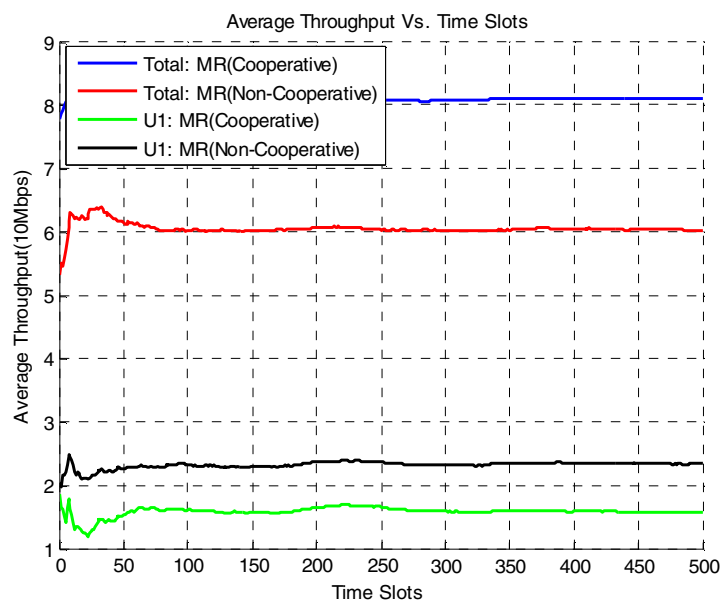

Fig. 1: The impact of selfish behavior on the throughput of packet scheduling algorithm.

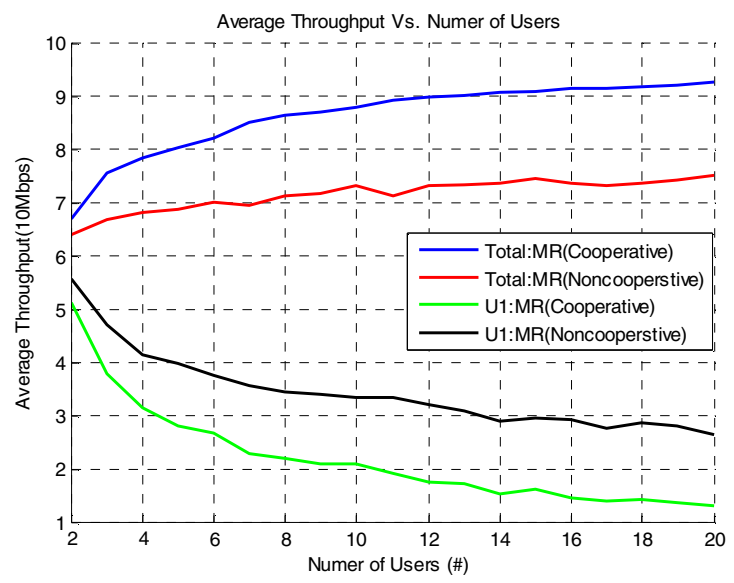

Fig. 2: The impact of selfish behavior on the throughput with different users in the system.

\section{Static GAme}

\section{A. Game model}

The above analysis clearly shows that the selfish user has an incentive to act in a non-cooperative way so as to improve its own throughput, and it can be modeled into a non-cooperative game in which each player has a continuum of actions [13]. In this game, we denote users $\mathbb{N}=\{1, \cdots, N\}$ as the set of players. When player $i$ 's maximal feasible rate is $r_{i}^{0}$, its set of actions is the interval from $r_{i}^{0}$ to $r^{M}$, i.e. $A_{i}=r_{i} \in\left[r_{i}^{0}, r^{M}\right]$. The action combination is denoted as $r=\left(r_{1}, r_{2}, \cdots, r_{N}\right) \in A$, where $A=\times_{i \in \mathrm{N}} A_{i}$ is the Cartesian product of the $N$ players' action profile. We identify each player's mixed strategy with a cumulative distribution function (CDF) $\quad F_{i}$ on this interval, for which $0 \leq F_{i}\left(r_{i}\right) \leq 1$ for every action $r_{i}$; and the number $F_{i}\left(r_{i}\right)$ is the probability that player $i$ 's data rate is at most $r_{i}$. Each player $i$ 's preference is represented by the expected value of the data rate:

$$
u_{i}(r)=\left\{\begin{array}{cc}
r_{i} \cdot \alpha\left(r_{i}, r_{i}^{0}\right) & r_{i}>r_{j} \\
1 / N \cdot r_{i} \cdot \alpha\left(r_{i}, r_{i}^{0}\right) & r_{i}=r_{j} \\
0 & r_{i}<r_{j}
\end{array}\right.
$$

where the parameter $1 / N$ means that when these players have the same data rates, they will be chose with the equal probability. We assume all user rates $r_{i}$ 's are independent and identically distributed with the same CDF, and then the expected payoff can be expressed as:

$$
\begin{aligned}
U_{\mathrm{i}}(r, F) \\
=r_{i} \cdot \alpha\left(r_{i}, r_{i}^{0}\right) \cdot \operatorname{Pr}\left(r_{i}>r_{j}\right)+ \\
\quad 1 / N \cdot r_{i} \cdot \alpha\left(r_{i}, r_{i}^{0}\right) \cdot \operatorname{Pr}\left(r_{i}=r_{j}\right)+0 \cdot \operatorname{Pr}\left(r_{i}<r_{j}\right) \\
=r_{i} \cdot \alpha\left(r_{i}, r_{i}^{0}\right) \cdot \operatorname{Pr}\left(r_{i}>r_{j}, \forall j \neq i\right) \\
=r_{i} \cdot \alpha\left(r_{i}, r_{i}^{0}\right) \cdot\left(F_{j}\left(r_{i}\right)\right)^{N-1}
\end{aligned}
$$

Within the above model, NE can be defined as follows. Definition 1: An action combination $r^{*} \in A$ and the corresponding mixed strategy $F^{*} \in[0,1]$ are said to achieve the state of Nash equilibrium if for every player $i \in \mathbb{N}$, we have:

$\forall r_{i}^{\prime} \in A_{i}, F_{i}^{\prime} \in[0,1], U_{i}\left(r_{i}^{*}, r_{-i}^{*} ; F_{i}^{*}, F_{-i}^{*}\right) \geq U_{i}\left(r_{i}^{\prime}, r_{-i}^{*} ; F_{i}^{\prime}, F_{-i}^{*}\right)$

where $r_{-i}$ and $F_{-i}$ denotes the actions and the corresponding mixed strategies chosen by everyone else other than $i$.

\section{B. Nash equilibrium}

For a game in which each player has finitely many actions, when a mixed strategy profile is a mixed strategy $\mathrm{NE}$, the expected payoff to every action assigned with possible probability is the same. Correspondingly, as described in the Proposition 142.2 in [13], for the game in which each user has infinitely many actions $A_{i}=\left\{r_{i} \mid r^{1} \leq r_{i} \leq r^{M}\right\}$, the mixed strategy is determined by the probabilities assigned to sets of actions; and the expected payoff should be constant from $r_{i}^{0}$ to $r^{M}$ in NE. Moreover, since $F_{j}\left(r_{i}=r^{M}\right)=1$, we have:

$$
\begin{aligned}
& U_{i}(r, F) \\
& =\left\{\begin{array}{cc}
r_{i} \cdot \alpha\left(r_{i}, r_{i}^{0}\right) \cdot\left(F_{j}\left(r_{i}\right)\right)^{N-1}=C & r_{i}^{0} \leq r_{i}<r^{M} \\
r^{M} \cdot \alpha\left(r^{M}, r_{i}^{0}\right) \cdot\left(F_{j}\left(r_{i}\right)\right)^{N-1}=C & r_{i}=r^{M}
\end{array}\right.
\end{aligned}
$$

where $\mathrm{C}$ is a constant. Thus, for $r_{i}^{0} \leq r_{i} \leq r^{M}, r^{1} \leq r_{i}^{0} \leq r^{M}$, we get:

$$
F_{j}\left(r_{i}\right)=\left(\frac{r^{M} \cdot \alpha\left(r^{M}, r_{i}^{0}\right)}{r_{i} \cdot \alpha\left(r_{i}, r_{i}^{0}\right)}\right)^{\frac{1}{N-1}}
$$

When the maximum achievable rate for player $i$ is $r_{i}^{0}$, it will choose its feedback rate $r_{i}$ according to the above NE strategy. Since in practice $r_{i}$ is discrete random variable, then if $r_{i}^{0} \leq r^{a} \leq r_{i} \leq r^{b} \leq r^{M}$, the probability that it report $r_{i}$ to BS would be expressed as:

$$
\operatorname{Pr}\left(r_{i}\right)=\frac{1}{2} \cdot\left(F_{i}\left(r^{b}\right)+F_{i}\left(r^{a}\right)\right)
$$




\section{Performance analysis}

In a cooperative environment, the average throughput of the system is maximized by MR algorithm. Furthermore, it is also Pareto efficient since it is impossible to make one user get a higher rate without adversely affecting other users. While in a non-cooperative environment, the selfish behavior breaks this property. With the same simulation environment in Section II, we plot the average system throughput for a network with 8 users in Fig.3. We can see that in this 8-player game, the average system throughput in NE state is much smaller than that in cooperative environment. This phenomenon follows readily from Fig. 4, where the network size varies from 2 users to 20 users. Thus the achieved NE throughput is Pareto inefficient, which is a common characteristic for a non-cooperative game.

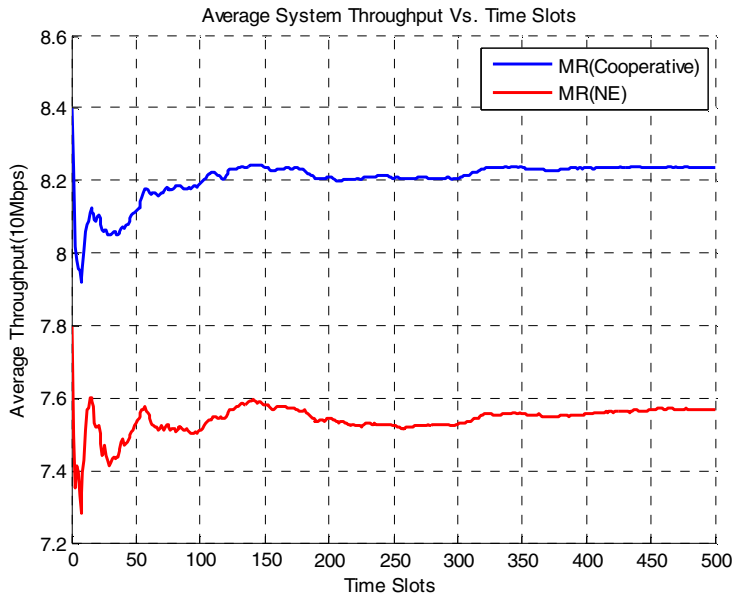

Fig. 3: Average system throughput comparison with 8 users under cooperative and NE conditions.

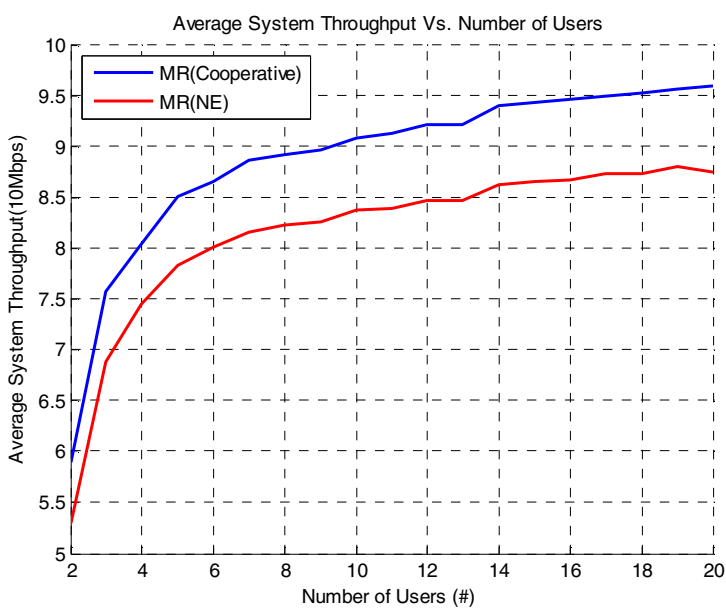

Fig. 4: Average system throughput with different users.

\section{REPEATED GAME}

The main reason for the throughput decrease in a non-cooperative environment is that the selfish user intents to report a higher data rate. Thus, if the user in a bad channel condition gives up the competition and lets the others to transmit, the throughput may be increased. However, in a non-cooperative environment, a rational user has little incentive to give up its channel if there is no mechanism to enforce cooperation.

In this section, we propose a repeated game to enforce cooperation. We assume that the users do not know the end of the game; hence we study the problem in an infinite repeated game model with discounting [13]. We show that cooperation (i.e., all users will report real $r_{i}^{0}$ instead of $r_{i}^{N E}$ with mixed NE strategy $\left.F_{i}\left(r_{i}\right)\right)$ can be enforced so as to improve the throughput for users.

\section{A. Repeated game model}

We extend the NPS game as follows: we assume that the game is split up steps denoted by $t$. In each step, user $i \in \mathbb{N}$ adjusts the rate according to its strategy. Furthermore, let us define the discounted average utility in $h<+\infty$ time steps as

$$
\overline{U_{i}}(h)=(1-\omega) \cdot \sum_{t=0}^{h} U_{i}(t) \cdot \omega^{t}
$$

where $0<\omega<1$ is the discounting factor, which can be interpreted as the probability that the game ends in the next step. And we assume that $\boldsymbol{\omega}$ is the same for all users.

We have found that the users are in an inefficient equilibrium when they all play NE strategy, whereas the maximal throughput can be achieved by playing cooperative strategy. From Folk Theorem [13], we know that in an infinitely repeated game, any feasible outcome that gives each player better payoff than the NE can be obtained. We now get conditions that enable the users to enforce cooperation, and prove that they can do better by applying a strategy called Striker.

\section{B. Striker strategy}

Definition 2: if user $i$ plays the Striker strategy, it plays $r_{i}^{0}$ in the first time step. For any further time steps, it plays:

- $\quad r_{i}^{0}$ in the next time step if the other player $j$ played $r_{j}^{0}$ in the previous time step, or

- $r^{M}$ for the next $h_{i}$ time steps, if the other played anything else.

The punishment interval $h_{i}$ defines the number of time steps for which player $i$ punishes the other players [15]. To simplify our analysis, we assume that the overall channel conditions remain relatively unchanged. Then, $r_{i}^{0}$ over each step in the repeated game is similar. However, our simulation results show that our analysis still holds in wireless fading situations. Then the cooperation can be enforced using the Striker strategy as formalized in the following proposition.

Proposition 1: An efficient Nash equilibrium can be enforced by the Striker strategy.

Proof: We consider the Striker strategy, and suppose player $i$ adhere to it and choose $r_{i}^{0}$.If player $j \neq i$ uses the same strategy, then the outcome is $\left(U_{i}^{C O P}, U_{j}^{C O P}\right)$ in every step, so that it obtains the stream of payoffs, which gives a discounted average of $(1-\omega) \cdot \sum_{i=0}^{h_{i}} U_{i}^{C O P} \cdot \omega^{t}=\left(1-\omega^{h_{i}+1}\right) \cdot U_{i}^{C O P}$.

If player $j$ adopts a price $r_{j}^{X}$ so as to get a larger utility $U_{j}^{N C O P}>U_{j}^{C O P}$ in subsequent steps, player $i$ will chooses $r^{M}$ since player $j$ 's choice of $r^{X}$ triggers the punishment. Then player $j$ chooses $r^{M}$ in every subsequent 
step with utility $U_{j}^{M}$. Consequently, it obtains the stream of payoffs with discounted average utility:

$$
\begin{aligned}
& (1-\omega) \cdot\left(U_{j}^{N C O P}+U_{j}^{M}+\omega \cdot U_{j}^{M}+\cdots+\omega^{h_{i}} \cdot U_{j}^{M}\right) \\
& =(1-\omega) \cdot U_{j}^{N C O P}+\left(1-\omega^{h_{i}+1}\right) \cdot U_{j}^{N E}
\end{aligned}
$$

Thus, player $j$ can not increase its utility by deviating if and only if:

$$
(1-\omega) \cdot U_{j}^{N C O P}+\left(1-\omega^{h_{i}+1}\right) \cdot U_{j}^{M}<\left(1-\omega^{h_{i}+1}\right) \cdot U_{j}^{C O P}
$$

Thus,

$$
\omega^{h_{i}+1}<1-(1-\omega) \cdot \frac{U_{j}^{N C O P}}{U_{j}^{C O P}-U_{j}^{M}}
$$

The inequality cannot be fulfilled if the right side is negative, and therefore:

$$
(1-\omega) \cdot \frac{U_{j}^{N C O P}}{U_{j}^{C O P}-U_{j}^{M}}<1
$$

When this condition holds, since $\omega<1$, we have:

$$
h_{i} \geq \log _{\omega}\left(1-(1-\omega) \cdot \frac{U_{j}^{N C O P}}{U_{j}^{C O P}-U_{j}^{M}}\right)-1
$$

Thus when the discounting factor is chosen as in (16) and punishment interval is set according to (17), the player $j$ will be forced to cooperate with others such as to get higher payoff by Striker strategy. Correspondingly, an efficient NE is achieved.

\section{Performance analysis}

To demonstrate the effect of Striker strategy, we perform simulations with 20 users. And the performance result shown is averaged over 20 channel and location realizations. The punishment interval is 10 time slots. User 1 deviated from the cooperative action at time slot 100. As shown in Fig.5, it can obtain a dramatic gain in its average throughput, which increased up to $13 \mathrm{Mbps}$. We can see that this defection was retaliated by other users in the system soon, which then made the throughput of this selfish user decrease dramatically. After that the throughput stabilized and returned to the cooperative state via Striker strategy. Thus a more efficient equilibrium is achieved as well as the scheduling performance is optimized in non-cooperative environment.

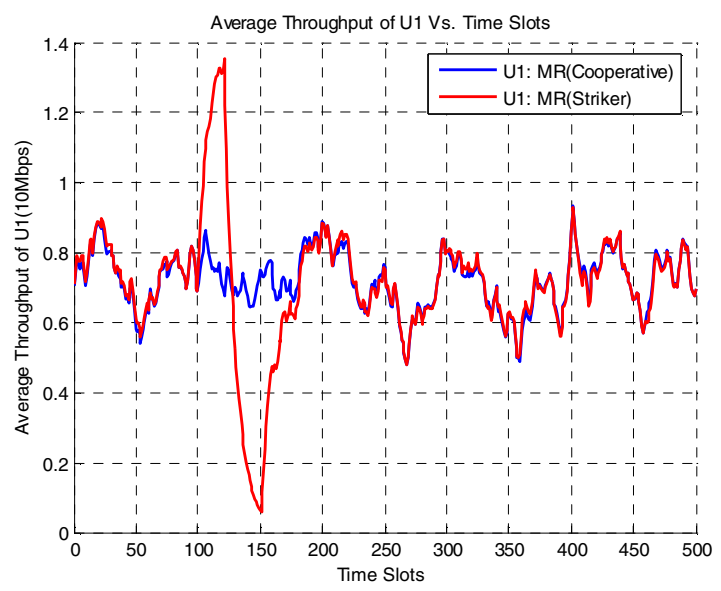

Fig. 5: Average throughput of User 1 under different strategies.

\section{CONCLUSIONS}

In this paper we investigate the impact of selfish and rational behavior on the throughput performance of MR packet scheduling algorithm in non-cooperative wireless networks. Our first contribution is that we set up a novel mixed strategy game model to analysis this problem and deduce the corresponding Nash equilibrium, in which the system throughput is found to be significantly reduced. Our second contribution is that we further propose a Striker strategy based on repeated game to enforce cooperation among selfish users and achieve a more desirable Nash equilibrium, in which the throughput performance can be increased. We are now analyzing the impact of selfishness on fairness-oriented packet scheduling algorithms in a non-cooperative environment.

\section{REFERENCES}

[1] B.S. Tsybakov, "File Transmission Over Wireless Fast Fading Downlink," IEEE Trans. Inf. Theory, vol. 48, Aug. 2002, pp. 2323-2337

[2] A. Jalali, R. Padovani, and R. Pankai, "Data Throughput of CDMA-HDR a High Efficiency-High Data Rate Personal Communication Wireless System," in Proc. Veh. Technol. Conf., Jan. 2001, pp. 55-67

[3] G. Tan and J. Guttag, "The 802.11 MAC Protocol Leads to Inefficient Equilibria," in Proc. IEEE INFOCOM'05, vol.1, March 2005, pp.1-11

[4] M. Raya, J. P. Hubaux, I. Aad, "Domimo: A System to Detect Greedy Behavior in IEEE 802.11 Hotspots", in Proc. ACM MoBiSys'04, June 2004, pp. 84-97

[5] S. Eidenbenz, G. Resta, P. Santi, "The COMMIT Protocol for Truthful and Cost-Efficient Routing in Ad Hoc Networks with Selfish Nodes", IEEE Trans. Mobile Computing, Vol. 7, Issue 1, Jan 2008, pp. 19-33

[6] W. Wang, X. Y. Li, "Low-cost routing in selfish and rational wireless ad hoc networks", IEEE Trans. Mobile Computing, vol. 5, May 2006, pp. $596-607$

[7] F. Meshkati, M. Chiang, H. V. Poor and S. C. Schwartz, "A Game-Theoretic Approach to Energy-Efficient Power Control in Multicarrier CDMA Systems," IEEE J. Select. Areas Commun., Vol. 24, June 2006, pp.1115 -1129

[8] S. Koskie, and Z. Gajic, "A Nash Game Algorithm for SIR-Based Power Control in 3G Wireless CDMA Networks," IEEE/ACM Trans. Networking, vol.13, Oct. 2005, pp.1017-1026

[9] M. Cagalj, S. Ganeriwal, I. Aad, J. P. Hubaux, "On Selfish Behavior in CSMA/CA Networks", in Proc. IEEE INFOCOM'05, vol.4, March 2005, pp.2513-2524

[10] P. Kyasanur and N. H. Vaidya, "Selfish MAC layer misbehavior in wireless networks,", IEEE Trans. Mobile Computing, Vol. 4, Sept.-Oct. 2005, pp. 502-516

[11] S. Radosavac, J. S. Baras, I. Koutsopoulos, "A Framework for MAC Protocol Misbehavior Dectection in Wireless Networks", in Proc. ACM WiSe'05, Sep. 2005, pp. 33-42

[12] P. P. C. Lee, T. Bu, T. Woo, "On the Detection of Signaling DoS Attacks on 3G Wireless Networks", in Proc. IEEE INFOCOM'07, 2007, pp.1289-1297

[13] M. J. Osborne, An Introduction to Game Theory, New York: Oxford University Press, 2004

[14] A.J. Goldsmith and S.G. Chua, "Variable-Rate Variable-Power M-QAM for Fading Channels", IEEE Trans. Commun., Oct. 1997, pp.1218-1230

[15] M. Felegyhazi, J. P. Hubaux, "Wireless Operators in Shared Spectrum", IEEE INFOCOM'06, 2006 